\title{
An Accurate Measurement-Based Power Consumption Model for LTE Uplink Transmissions
}

\author{
Bjoern Dusza*, Christoph Ide*, Liang Cheng ${ }^{\dagger}$ and Christian Wietfeld* \\ ${ }^{*}$ Communication Networks Institute, TU Dortmund University, 44227 Dortmund, Germany \\ e-mail: \{Bjoern.Dusza, Christoph.Ide, Christian.Wietfeld\}@tu-dortmund.de \\ ${ }^{\dagger}$ Department of Computer Science and Engineering, Lehigh University, Bethlehem, PA, USA \\ e-mail: Cheng@cse.lehigh.edu
}

\begin{abstract}
In this poster the results of an accurate, measurement-based power consumption profiling of Long Term Evolution (LTE) User Equipment (UE) are presented together with concrete model parameter sets for four different most recent LTE data sticks and one LTE smart phone. In contrast to existing power consumption models for cellular devices, the model presented in this poster allows for a precise quantification of energy supply requirements depending on the LTE uplink data transmission power, the device type and the carrier frequency. This knowledge can be for example applied for the suitable dimensioning of batteries and energy harvesting devices in the context of LTE-based wireless sensor networks.
\end{abstract}

\section{Motivation}

The battery lifetime is one of the most important performance parameters for customers. Also in the context of LTEenabled remote sensing, energy consumption is a topic of major importance. The communication activities -in particular the uplink transmissions- are one of the major drivers of the overall power consumption. The important relationship between the uplink transmission power $P_{T x}[\mathrm{dBm}]$ and the overall power consumption of the UE $\bar{P}$ [W] has however not been sufficiently addressed for LTE so far. Especially for

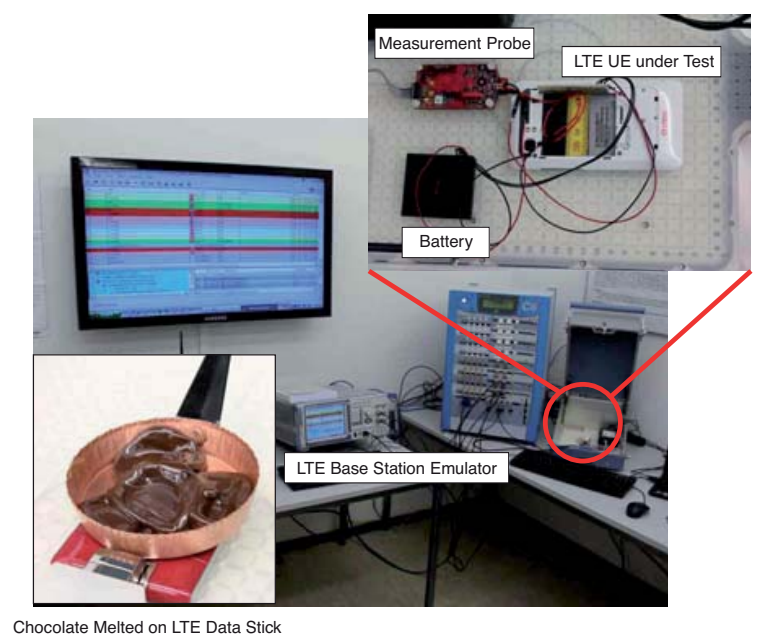

Fig. 1. Lab Facilities for Power Consumption Measurements. high transmission powers, the LTE UE platform consumes a disproportional amount of power leading to increased heat loss. This effect is illustrated by an experiment shown in Fig. 1. The picture (left bottom) shows chocolate which was melted after 45 min of continuous operation of an LTE stick at the maximum allowed transmission power of $23 \mathrm{dBm}$.

\section{EMpirical Power Consumption Modeling}

To derive an accurate power consumption model, extensive measurements for different commercially available LTE UEs have been performed in our mobile communications lab. Fig. 1 illustrates the measurement setup, which includes an LTE base station emulator to allow for an accurate control of the uplink transmission power of the LTE UE (cf. [1] for more details). The measured correlation between the uplink transmission power and the power consumption of the UE is illustrated in Fig. 2 for different devices operating in LTE band 7 (2.6 GHz).

One can see from the plot that the power consumption curve can be divided into two parts: For a low transmission power, below a device specific threshold $\gamma$, the graph is characterized by a small, almost horizontal slope. For higher transmission power values, the power amplifier switches the mode and the slope becomes significantly steeper. This specific characteristic can be observed for all UEs under test. The empirically derived power consumption curve $\bar{P}$ can be approximated by two

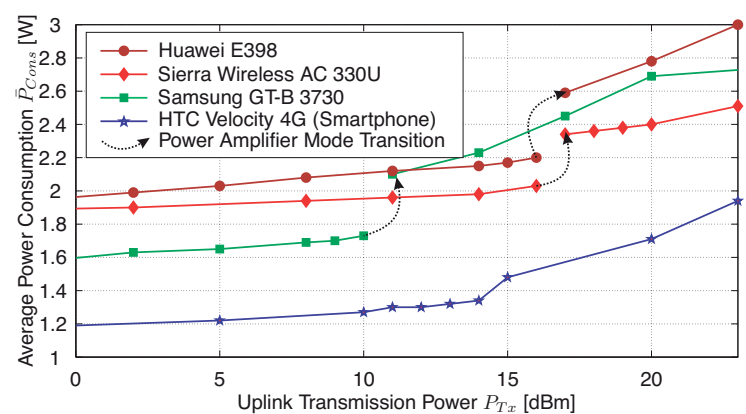

Fig. 2. Device-specific Power Consumptions for Different Uplink Transmission Power Values (all in $2.6 \mathrm{GHz}$ ). The results will be updated continuously as new LTE devices become available and are publicly accessible at www.cni.tu-dortmund.de/LTEpowermodel.

(c) 2013 IEEE. Personal use of this material is permitted. Permission from IEEE must be obtained for all other uses, in any current or future media, including reprinting/republishing this material for advertising or promotional purposes, creating new collective works, for resale or redistribution to servers or lists, or reuse of any copyrighted component of this work in other works.

The contribution will also be available at http://ieeexplore.iee.org 
TABLE I

EMPIRICAL MOdEL PARAMETERS FOR DifFERENT LTE UES.

\begin{tabular}{|c|c|c|c|c|c|c|c|}
\hline $\begin{array}{c}\text { Model } \\
\text { Parameter }\end{array}$ & \multicolumn{2}{|c|}{$\begin{array}{c}\text { HTC } \\
\text { Velocity 4G }\end{array}$} & $\begin{array}{c}\text { Samsung } \\
\text { GT-B } \\
3740\end{array}$ & $\begin{array}{c}\text { Samsung } \\
\text { GT-B } \\
3730\end{array}$ & $\begin{array}{c}\text { Huawei } \\
\text { E 398 }\end{array}$ & \multicolumn{2}{c|}{$\begin{array}{c}\text { Sierra Wireless } \\
\text { AC 330U }\end{array}$} \\
\hline Frequency [MHz] & 800 & 2600 & 800 & 2600 & 1800 & 2100 & 2600 \\
\hline$\alpha_{L}[\mathrm{~mW} / \mathrm{dBm}]$ & 4.8 & 4 & 7.7 & 7.2 & 10 & 5.6 & 5.4 \\
\hline$\beta_{L}[\mathrm{~W}]$ & 1.6 & 1.2 & 1.6 & 1.6 & 1.7 & 1.6 & 1.9 \\
\hline$\alpha_{H}[\mathrm{~mW} / \mathrm{dBm}]$ & 68 & 61 & 13 & 54 & 24 & 27 & 28 \\
\hline$\beta_{H}[\mathrm{~W}]$ & 0.79 & 0.52 & 0.4 & 1.5 & 1.9 & 1.5 & 1.8 \\
\hline$\gamma[\mathrm{dBm}]$ & 12 & 12 & 11 & 10 & 16 & 16 & 16 \\
\hline $\bar{P}_{I D L E}[\mathrm{~mW}]$ & 40 & 40 & 175 & 44 & 236 & 63 & 63 \\
\hline Maximum Error [\%] & 5.1 & 3.5 & 1.7 & 3.9 & 4.7 & 3.6 & 1.5 \\
\hline
\end{tabular}

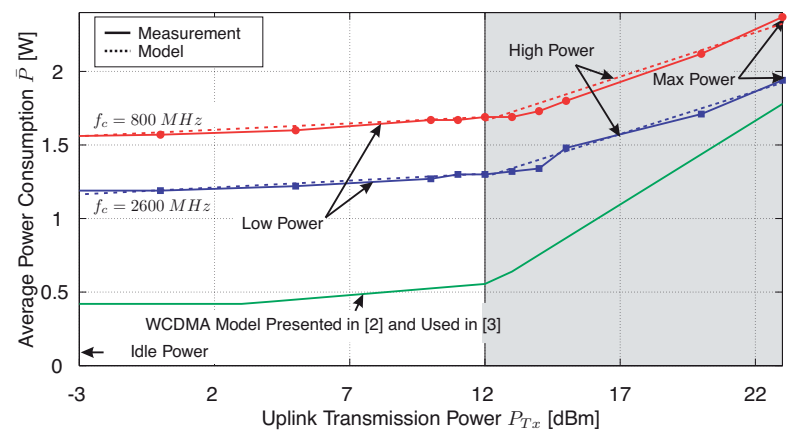

Fig. 3. Tx-Power Dependent Average Power Consumption vs. Empirical Model (HTC Velocity 4G, cf. Table I).

linear functions:

$$
\bar{P}\left(P_{T x}\right)= \begin{cases}\alpha_{L} \cdot P_{T x}+\beta_{L} & \text { for } P_{T x} \leq \gamma \\ \alpha_{H} \cdot P_{T x}+\beta_{H} & \text { for } P_{T x}>\gamma\end{cases}
$$

with the device specific parameters $\alpha, \beta$ and $\gamma$ as given in Table $\mathrm{I}$ and the uplink transmission power $P_{T x}$. For the downlink reception our measurements have shown that the consumed power is practically independent of downlink system parameters and can be approximated by the $\beta_{L}$ value.

Table I provides the approximation error for all devices. As an example, Fig. 3 illustrates in detail the good match between the proposed model and the actual measurements for the HTC velocity $4 \mathrm{G}$ smartphone and both frequency bands supported by this device. Additionally, the power consumption model for the WCDMA UE presented in [2] and used in [3] for LTE devices is shown. Comparing the curves one can clearly observe that the previous work significantly underestimates the power consumption of the UE (by $0.75 \mathrm{~W}$ in case of $12 \mathrm{dBm}$ uplink transmit power).

\section{EXAmple ApPlication: Dimensioning OF ENERGy STORAGE FOR WirelESS SENSOR NODES}

One application of the proposed LTE power consumption model is the suitable dimensioning of batteries and energy harvesting devices for wireless sensor networks (e.g. for video surveillance in emergency situations when the grid or the power connection to grid is destroyed). Assuming that a certain amount of data must be transferred before the battery runs out, the energy $E_{B i t}$ that needs to be spent for the transmission of one bit is used to determine the suitable size of the energy

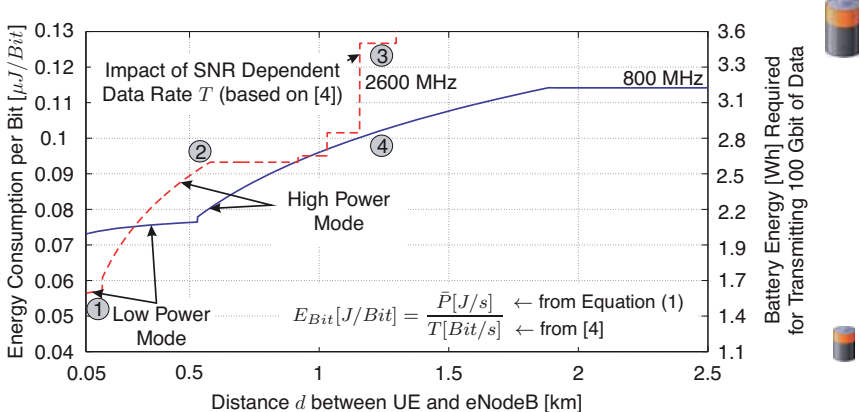

Fig. 4. Energy Consumption per Bit $E_{B i t}$ and Battery Energy Requirement vs. Communication Distance $d$ for Wide Area Wireless Sensing Applications (assuming free space propagations losses).

storage. This figure of merit can be calculated by dividing the power consumption $\bar{P}\left(P_{T x}\right)$ by the throughput $T$ (cf. formula in Fig. 4). Both parameters depend on the distance $d$ between $\mathrm{UE}$ and eNodeB. We assume that a target Signal to Noise Ratio (SNR) of $30 \mathrm{~dB}$ is required at the base station for achieving the maximum throughput. An increase of the distance $d$ therefore leads to an increased path loss and a respectively higher transmission power $P_{T x}$ that is required for compensating it. While $\bar{P}$ depends on $P_{T x}$ (cf. Equation (1)) the throughput $T$ is a function of the SNR as shown in [4]. For high distances the path loss can no longer be compensated by adjusting $P_{T x}$ (due to the limitation of the transmission power to $23 \mathrm{dBm}$ ) which leads to a lower $T$ and therefore a higher $E_{B i t}$. Fig. 4 illustrates $E_{B i t}$ vs. the distance $d$ for two frequently used LTE frequency bands. Beyond that, the energy per bit is translated to a distance dependent battery capacity that is as least required for transmitting $100 \mathrm{Gbit}$ of data. One can observe that for the case of $2.6 \mathrm{GHz}$ increasing the communication distance from $50 \mathrm{~m}$ to $500 \mathrm{~m}$ requires a $59 \%$ larger energy storage (cf. (1) and (2) in Fig. 4). For a distance of $1250 \mathrm{~m}$, using LTE at $800 \mathrm{MHz}$ instead of $2.6 \mathrm{GHz}$ allows for battery capacity savings of $20 \%$ (cf. (3) and (4) in Fig. 4). This is because the lower path loss at $800 \mathrm{MHz}$ overcompensates the lower efficiency. For higher communication distances, the achievable gain by using an $800 \mathrm{MHz}$ system is further increasing.

\section{ACKNOWLEDGMENT}

Part of the work on this paper has been supported by Deutsche Forschungsgemeinschaft (DFG) within the Collaborative Research Center SFB 876 Providing Information by Resource-Constrained Analysis, projects A4 and B4.

\section{REFERENCES}

[1] B. Dusza et al., "Utilizing Unused Network Capacity for Battery Lifetime Extension of LTE Devices", Proc. IEEE Int. Conf. on Communications (ICC) Workshops, Ottawa, Canada, 2012.

[2] H. Holma, A. Toskala, "WCDMA for UMTS - HSPA Evolution and LTE", 5th ed., John Wiley \& Sons, Ltd., 2010.

[3] M. Lauridsen et al., "Reducing LTE Uplink Transmission Energy by Allocating Resources", Proc. IEEE 74th Vehicular Technology Conference Fall (VTC-Fall), San Francisco, CA, USA, 2011.

[4] B. Dusza et al., "Measuring the Impact of the Mobile Radio Channel on the Energy Efficiency of LTE User Equipments", Proc. 21st Int. Conf. on Computer Communication Networks (ICCCN), Munich, Germany, 2012. 